\title{
INTELLIGENT METAL POWDER LASER FORMING SYSTEM
}

\author{
Fei Xing ${ }^{1,2}$, Weijun Liu ${ }^{1}$, Kai Zhang ${ }^{1,2}$, Xiaofeng Shang ${ }^{1}$,Tianran Wang ${ }^{1}$ \\ ${ }^{\prime}$ Advanced Manufacture Lab, Shenyang Institute of Automation, CSA, Shenyang, Liaoning \\ Province, China; E-mail: xingfei@sia.cn. ${ }^{2}$ Graduate School, Chinese Academy of Sciences, \\ Beijing, China.
}

\begin{abstract}
Metal Power Laser Forming (MPLF) is a high precision and complicated process. The quality of the formed parts is affected by many parameters and may vary significantly during the laser forming process. In order to guarantee the stabilities of the process, a state-of-the-art intelligent MPLF system was developed in this paper. The intelligent system includes CNC system, energy supply and control system, powder delivering and control system, and realtime feedback control system. The established state-of-the-art apparatus and several metal parts formed by the MPLF system are presented in this paper.
\end{abstract}

Key words: Metal Power Laser Forming, monitor, control, laser cladding, apparatus.

\section{INTRODUCTION}

Metal Power Laser Forming (MPLF) is an advanced laser materials processing technique, which is widely used in manufacturing, part reparation, surface modification, and Solid Freeform Fabrication (SFF). In the MPLF process, the metal powder sprayed form the powder delivery nozzle and injected into the laser focal zone, together with a thin layer of the moving substrate, is melted by a laser beam and deposited a layer on the substrate [1]. A great variety of materials can be deposited on a substrate to form a layer with a thickness ranging from 0.1 to $2 \mathrm{~mm}$. This technique can produce a much better coating, with minimal dilution, minimal distortion, full-density and good surface quality than other techniques such as arc welding and plasma spraying. These advantages have recently been found attractive in industries for tools manufacturing and metallic rapid prototyping [2]. This

This project was financially supported by the National " 863 " High-technology Research Project (Grants No. 2002AA420060) and the Knowledge Innovation Project of the Chinese Academy of Science (Grants No.F040208).

Please use the following format when citing this chapter.

Xing, Fei, Liu, Weijun, Zhang, Kai, Shang, Xiaofeng, Wang, Tianran, 2006, in International Federation for Information Processing (IFIP), Volume 207, Knowledge Enterprise: Intelligent Strategies In Product Design, Manufacturing, and Management, eds. K. Wang, Kovacs G., Wozny M., Fang M., (Boston: Springer), pp. 525-535. 
technique is also considered as the best way for manufacturing of functionally-graded parts.

Similar to other rapid prototyping techniques, in rapid prototyping using laser forming, a three-dimensional (3D) CAD solid model is used to produce a part without intermediate steps. This approach to produce a mechanical component in a layer-by-layer fashion allows us to fabricate a part with features that may be unique to laser cladding prototyping. These features include a homogeneous structure, enhanced mechanical properties, and production of complex geometries. However, the clad quality may vary significantly during a laser cladding process. Variations of the quality may even be observed between processing cycles performed using the same operating conditions. This poor reproducibility arises from the high sensitivity of laser cladding to small changes in the operating parameters such as laser power, beam velocity and powder delivery rate, as well as to process disturbances such as variations in absorptivity [3]. As a result, development of an intelligent MPLF system is essential for overcoming the effects of disturbances in the process.

At present an advanced intelligent MPLF system is successfully established at Shenyang Institute of Automation Chinese Academy of Sciences. In this paper, the components of the intelligent MPLF system are explained in detail, and several metal parts formed by the LMDS system are presented.

\section{THE INTELLIGENT MPLF SYSTEM}

The intelligent MPLF system includes CNC system, energy supply and control system, powder delivery and control system, and real-time feedback control system. It can fabricate fully dense, highly accurate complex parts and tools directly from a CAD solid model. Firstly a 3D CAD solid model is sliced into many layers and produces a CLI (Common Layer Interface) files by the system application software. The software also provides a graphical user interface (GUI) for the control of CAD data processing, the motion of the stage, and the powder regulation, as well as the controls of the safety and processing shutters. The laser head and the worktable governed by the CNC system move together to insure that the injected powder is melted onto the substrate as the laser scans over the part. Sensing and control systems are developed to guarantee the stabilities of the process, such as the stability of the melt pool's temperature and shape, the stability of the powder delivery rate, the stability of the single cladding's height. The real photograph and schematic illustration of the MPLF system are presented respectively in Fig. 
1(a) and 1(b). The detail explanation of the developed MPLF apparatus will be individually presented as follows.

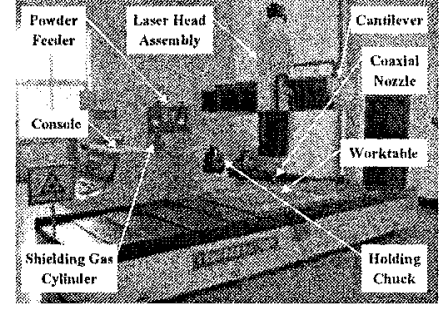

(a) Real photograph of MPLF system.

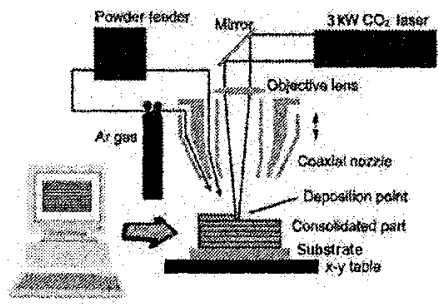

(b) Schematic of the MPLF system.

Figure 1. The intelligent Metal Powder Laser Forming System.

\subsection{CNC system}

The CNC system consists of industrial computer, several motors, motion control cards, $\mathrm{NC}$ worktable, system application software, and corresponding circuits. It mainly includes a six-axis NC machine tool, namely three precise controlled linear axes (i.e. $\mathrm{X}, \mathrm{Y}, \mathrm{Z}$ ) and three free rotation axes that rotate around the $\mathrm{X}, \mathrm{Y}$, and $\mathrm{Z}$ axes individually. The machine tool can achieve four-axis linked motion by the control of the industrial computer.

Two sets of PCI-1240 motion control cards are installed in the computer. The NC worktable is communicated with the industrial computer through one control card. Two motors of the powder feeders are governed by the other control card. During the MPLF process, the X-Y plane worktable achieves the horizontal motion of substrate and deposited material. The powder nozzle head is mounted on a vertical linear Z-axis of the $\mathrm{NC}$ worktable. The $Z$-axis provides the vertical motion of the nozzle head during the deposition process. The positions and velocities of $X, Y$, and $Z$ axes are regulated via the $\mathrm{PCI}-1240$ motion card by the industrial computer. Each linear axis is equipped with two limit switches which restrict its own motion range in order to ensure the safety and stability of the MPLF system. From Table.1 we can see that the system has good accuracy and repeatability.

Table 1. The performance of the CNC system

\begin{tabular}{ll}
\hline Motion range of the worktable & $2000 \mathrm{~mm} \times 1000 \mathrm{~mm} \times 500 \mathrm{~mm}$ \\
\hline Accuracy of X,Y,Z axis & $\pm 0.08 \mathrm{~mm}, \pm 0.08 \mathrm{~mm}, \pm 0.05 \mathrm{~mm}$ \\
\hline Repeatability & $\pm 0.05 \mathrm{~mm}$ \\
\hline
\end{tabular}

However, the exact degree of repeatability in this MPLF equipment will vary from table to table but in comparison with the variability associated with the laser and the material it is unlikely to be a significant factor. At the 
same time a laser forming system based entirely on predictive and theoretical work would be impractical. A better approach to achieve precision laser forming would be to introduce on-line measurement to continually monitor the process and control key parameters rather than rely entirely on predictive information.

\subsection{Energy supply and control system}

The laser power supply system is a console mounted energy supply with a cooling system. A crosscurrent, pipe sheet, multimode, $3 \mathrm{~kW} \mathrm{CO} 2$ LASER, whose wavelength is $10.6 \mu \mathrm{m}$, is employed in the MPLF system as the power source.

The parameters of the energy supply system are the laser power, beam diameter, beam mode structure, and beam focal point. The effective energy (the amount of energy delivered to the substrate) depends on the laser power, CNC scanning speed, and beam diameter and is expressed as $\mathrm{E}=\mathrm{P} /(\mathrm{Dv})$ where $E$ is the specific energy $\left(\mathrm{J} / \mathrm{mm}^{2}\right), v$ is the CNC traverse speed $(\mathrm{mm} / \mathrm{s})$, $\mathrm{P}$ is the laser power (W), and D is the spot size $(\mathrm{mm})$. Thus, in order to regulate the amount of energy delivered to the substrate, the laser power, beam diameter, and traverse speed have to be adjusted. A power collecting sensor is installed to monitor the laser power, which is displayed simultaneously. A feedback hardware system is developed to adjust the laser power. The feedback instruction sent from the computer via a PC RS232 serial port and an adjusting circuit is used to ensure optimum working electric current. Besides the high-energy LASER, the laser energy supply system includes light gate, calibration laser, reflector, plano-convex lens, and cooling system, as shown in Fig. 2 [4]. During deposition, the light gate, the calibration laser and reflector move together. A semiconductor laser, whose wavelength is $0.633 \mu \mathrm{m}$, emits the red calibration laser. The calibration laser and the $\mathrm{CO}_{2}$ laser are concentric. By real-time control of the laser power, many other process parameters, such as the temperature and geometry of the melt pool, can be optimized.

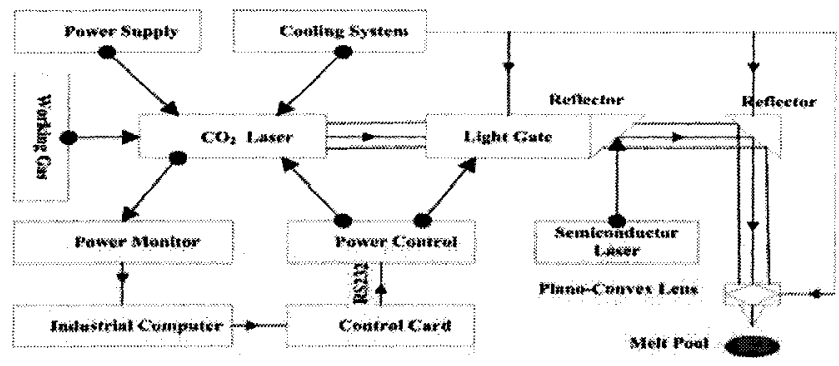

Figure 2. Schematic of the energy supply and control system 


\subsection{Powder delivery and control system}

The stability of the powder delivery speed is one of the most important parameters affecting the quality of the deposited parts. The controllable powder delivery can greatly improve the performance of 3D laser cladding. It also provides the feasibility to build a functional graded material or alloy.

There are two types of powder supply patterns, which are coaxial and lateral powder nozzle. However, when lateral powder nozzle is used, the metal powder can only be injected in one direction. 3D metal powder laser forming mainly depends on the directions of the powder delivery and the worktable movement. In order to solve the problem, a coaxial nozzle is invented to insure the metal powder is injected in the direction of the laser beam, and has been applied into the MPLF system. The real photo and sectional view of the coaxial powder nozzle is individually shown in Figs. 3(a) and 3(b). The powder is fed from four sides of the nozzle and uniformly distributed between powder taper sleeve and cooling water taper sleeve. The nozzle end has been specifically designed to enable the powder to be delivered out at a specific angle to the vertical so that the focal point of the powder coincides with the focal point of the laser beam. This allows maximum powder utilization during deposition. Moreover, the powder flow angle can be regulated by varying the shielding gas flow rates. Indispensably, water-cooling system has been well designed to keep the appropriate operating temperature for nozzle. Since the powder is injected directly to the molten pool, the powder nozzle can move in any directions on the surface. It makes the MPLF system process a high degree of flexibility and efficiency.
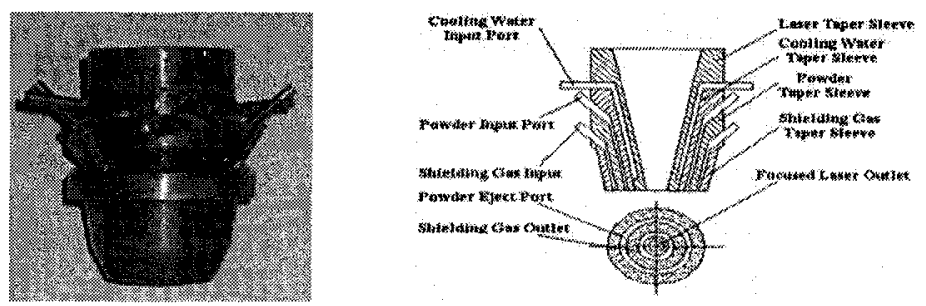

(a) Real photo of coaxial powder nozzle. (b) Sectional view of coaxial powder nozzle.

Figure 3. Coaxial powder nozzle of the MPLF system

Besides the coaxial powder nozzle, two powder feeding chambers is designed, with two individual powder hopper units assembled together, as shown in Fig.4(a). The powder nozzle and the powder feeding chambers together make up of the powder delivery system. Each powder hopper can hold a significant portion of the powder. During the deposition, the powder flows from the hopper outlet down to an annular groove of a rotary table in a 
chamber, as shown in Fig. 4(b). With the rotation of the disk, the powder in the annular groove is transported to a nearby suction nozzle, which is fixed over the annular groove, but a little space is left between them. By virtue of the air pressure of carrier gas, the powder is forced into the suction nozzle, and then is delivered to the nozzle head along the powder feeding tube. Each powder feeder is driven by a motor, and also has its own feeding tube. The two feeding tubes are connected into a mixing channel through a three-way coupling joint. The converged tube is subsequently divided into four parts by splitters, which uniformly distribute the powder into four tubes leading to the nozzle end. Then the powder is injected into the laser and generated melt pool. When two different kinds of powders are delivered, they can be mixed on line after they pass through the three-way coupling joint. By regularly adjusting the rotational speed of each motor, the functionally graded material can be fabricated freely.

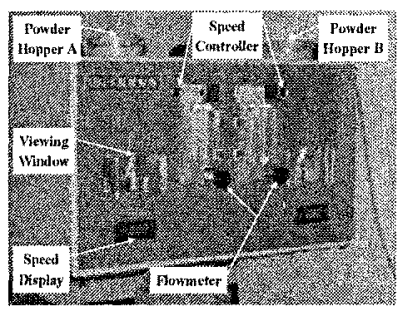

(a)

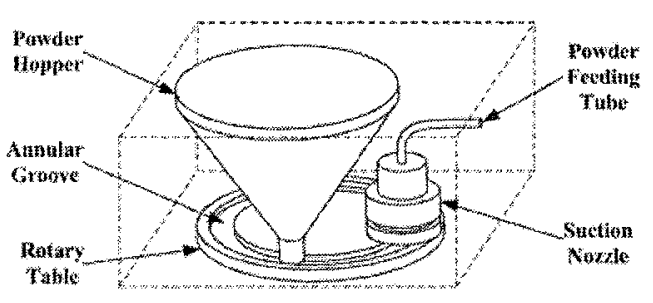

(b)

Figure 4. Powder feeding chambers of the MPLF system. (a) Real photo of powder feeding chambers. (b) Schematic of inner structure of powder feeding chamber.

In order to adjust the delivery speed of the metal powder, an optoelectronic sensor is developed to sense the real-time powder rate, as shown in Fig. 5(a). The sensor consists of a laser diode, a photo diode, and a glass window. The components are installed in such a way that the laser beam emitted from the laser diode passes through the powder stream flowing inside the glass chamber and is received by the photo diode. The carrier gas and the metal powder are mixed well, so the powder particles distribute uniformly in the carrier gas. Because of the diffusion, absorption, and reflection of the powder particles to the laser beam, the laser energy received by the photo diode decreases if the powder delivery rate increases, which means there is a higher percentage of powder particles in the carrier gas [5].

An on-line electronic scale is used to calibrate the sensor. The averaged powder delivery rate is calibrated by measuring the time period and the weight of the powder delivered during the time period. The corresponding sensing signal from the sensor is also acquired by a data acquisition card installed on the industrial computer. A linear relationship between the 
powder delivery rate and the averaged sensor output is established, as shown in Fig. 5(b). Then we can acquire the delivery rate according to the sensor output. A PID controller is developed to control the powder feed motor based on the delivery rate and the cladding height. The delay time of the powder delivery is measured beforehand in the control system [6].

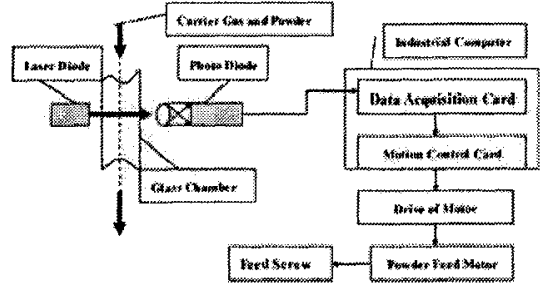

(a) Schematic of the feedback control system. (b) The performance of the sensor.

Figure 5. Metal powder monitoring and control system

\subsection{Real-time feedback control system}

As addressed in above sections, close monitoring and control of the variables of these systems are required to enhance the parts quality. Feedback controllers have to be designed to regulate these variables mainly to control melt pool size, temperature distribution in the melt pool, and cladding height and width. These variables may vary during the operation due to fluctuations in system parameters such as powder delivery rate, beam position and diameter, output power, and scanning speed, and presetting these operating parameters are not appropriate. They have to be monitored and optimized continuously to obtain the desired conditions.

\subsubsection{Feedback control system of the melt pool}

Temperature is a critical factor that requires continuous monitoring and control. It determines the melt pool dimensions and, hence, the dilution. If the temperature is too low, the resulting melt pool catches little powder and if the temperature is too high, it may melt back the substrate.

During the MPLF process, the temperature of the melt pool can reach $2500 \mathrm{~K}$ while high melting-point powder is injected, so non-contact measurement technique should be chosen to monitor the features of the melt pool. Colorimetric technique is developed to obtain the 2D temperature distributions of the melt pool [7]. The melt pool is sensed by a high framerate camera installed at the side of laser head, which takes grey images with a $640 \times 480$ resolution. The infrared radiation from melt pool produced by the 
laser beam passes through the lens and forms an image on the CCD chip of the camera.

In terms of the light path structure, the sensor is installed in the form of single passage, which signifies only one CCD sensor is used in the measuring system, as shown in Fig. 6(a). Two kinds of optical filters, which respectively capture infrared ray with specific wavelengths $(790 \mathrm{~nm}$ and $921 \mathrm{~nm}$ ), are fixed on a turnplate. With the rotation of the turnplate, the filters are put into the way of the light alternately. Since a step motor is used as driver and a photoelectric switch is used to ensure the position of the filters, the turnplate keeps fast rotary speed and accurate fix position. Considering the thermal inertial of the melt pool, two images of the melt pool are obtained in so little time by the high frame-rate camera that the two images can be regarded as being taken at the same time. Then we can acquire the temperature field and the shape of the melt pool through the colorimetric technique. As mentioned above, the features of the melt pool are greatly judged by the laser power and the scanning speed. Fig. 6(b) shows a fuzzy logic controller which is designed to maintain the stability of the melt pool by adjusting the inputs of the scanning speed and laser power based on the feedbacks [8].

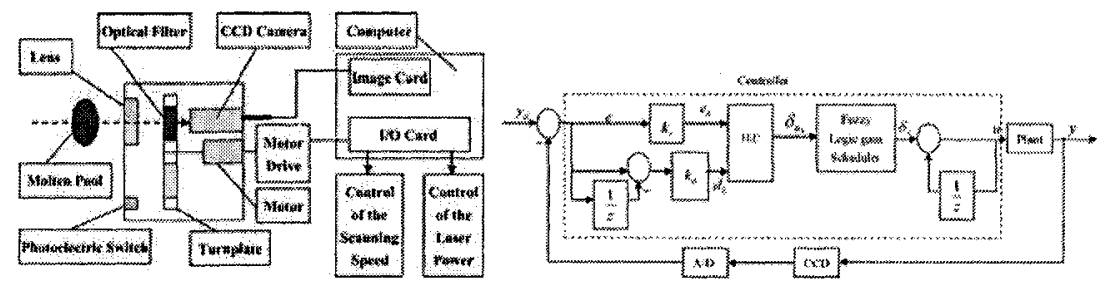

(a) Schematic of the feedback control system of melt pool. (b) Fuzzy logic controller.

Figure 6. Feedback control system of melt pool.

\subsubsection{Feedback control system of the cladding}

The dimensional accuracy of the parts depends on the uniformity and repeatability of the clad height and width being deposited. The cladding geometry is another important factor that requires continuous sensing and control. The feedback control system can not only improve the precision of the formed parts, but also save costs for the post treatment of the parts.

The monitoring and control system is constructed by an industrial CCD camera, a line-structure LASER, an industrial computer, an image card, and system software. Fig. 7(a) shows the schematic diagram of the monitoring system of the cladding. The CCD camera and the line-structure laser are installed at different sides of the laser head. A line laser emitting from the 
line-structure LASER forms a line on the surface of the metal part. At the same time the camera takes photos of the part, which are grabbed by the image card and transmitted to the computer. The program designed beforehand in the computer would manipulate these images, calculate the three-dimensional coordinates of the image points based on the world reference frame, and obtain the feedback of the height. The computer program on calculation of the cladding height includes four primary steps: (1) calibration of the camera and the line laser plane [9]; (2) calculation of the three-dimensional coordinates of the image points based on the camera reference frame; (3) calculation of the relational matrix between the camera reference frame and the world reference frame; (4) acquisition of the corresponding points of different claddings and calculation of the height. A feedback is acquired by comparing the actual height with the presetting height of the cladding layer. Besides the height of the cladding, the width can also be acquired by the CCD camera. As mentioned above, A PID controller is designed to adjust the powder feed motor in order to guaranteeing the stability of the cladding height. Fig.7 (b) shows the schematic of the feedback control system of the cladding.

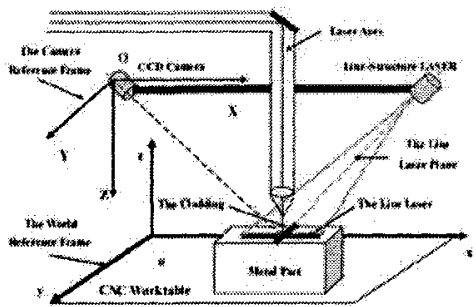

(a) Schematic of the monitoring system.

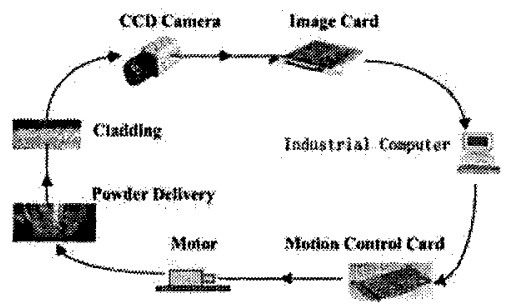

(b) Schematic of the feedback control system Figure 7. Monitoring and control system of the cladding

\section{METAL PARTS FORMED BY THE INTELLIGENT MPLF SYSTEM}

Base on intelligent MPLF system mentioned above, multilayered claddings were successively made to explore the feasibility of the fabrication of metal parts. With the optimal processing parameters ascertained by previous experiments and listed in Table 3, several fully-dense nickel-based metal parts were formed by the MPLF system, four of which are shown in Fig. 8. All of them demonstrate fine appearances and good surface quality. Besides, they are free of porosity, fully-dense, and near-net shaped. The density of these metal parts is expected to be the same as the parts fabricated 
by conventional manufacturing processes, because each layer was fused with metallurgical bonding and is free of porosity. In addition, rapid solidification during deposition ensures the fine microstructure and perfect properties of the fabricated metal parts.

Table 2. The optimal processing parameters of the MPLF system

\begin{tabular}{ccccc}
\hline $\begin{array}{c}\text { Laser power } \\
(\mathrm{W})\end{array}$ & $\begin{array}{c}\text { Scanning velocity } \\
(\mathrm{mm} / \mathrm{s})\end{array}$ & $\begin{array}{c}\text { Spot diameter } \\
(\mathrm{mm})\end{array}$ & $\begin{array}{c}\text { Powder delivery } \\
\text { rate }(\mathrm{g} / \mathrm{mm})\end{array}$ & $\begin{array}{c}\text { Scanning space } \\
(\mathrm{mm})\end{array}$ \\
\hline 1000 & 5 & 2 & 10 & 1.3 \\
\hline
\end{tabular}

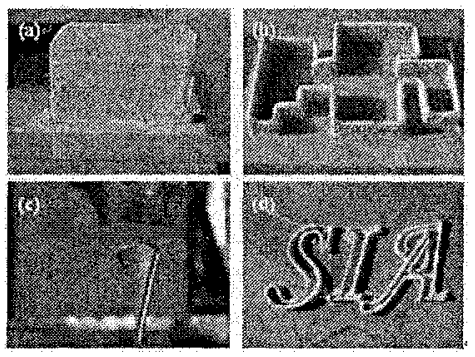

(a) Thin-wall sample. (b) Complex polygon sample. (c) Rotary blade sample. (d) Acronymic emblem of Shenyang Institute of Automation.

Figure 8 . Nickel-based metal parts formed by intelligent MPLF system

\section{CONCLUSIONS}

In this paper, an intelligent MPLF system is introduced. The detailed design and components of the MPLF system is presented, and its performance is evaluated. A CNC system is established to guarantee the accuracy and repeatability of the MPLF system. From experiments we find the performance of the system is good. A $3 \mathrm{~kW} \mathrm{CO} 2$ LASER is employed as the power source. The laser power is acquired and displayed by a power collecting sensor, and can be adjusted by a control card and an adjusting circuit. Powder supply and control system plays an important role in MPLF process. The design of the coaxial nozzle makes metal parts 3D fabrication possible, and the design of a state-of-the-art powder feeding apparatus makes that the functionally graded material parts can be fabricated freely. An optoelectronic sensor is developed to monitor the metal powder speed, which can be adjusted via the powder feed motor by the industrial computer and motion control card. The real-time sensing and control systems of the powder delivery speed and the laser power indicate feasible methods to guarantee the stability of the important process parameters, such as the 
temperature and geometry of the melt pool, the height and width of the cladding.

The process parameters need to be monitored and optimized continuously. Two feedback control systems of the melt pool and cladding, which are two critical factors of the MPLF process, are developed in this paper. Machine vision systems, based on industrial CCD cameras and computer, are used to sense the forming process. Colorimetric technique, a non-contact measurement technique, is developed to obtain the 2D temperature distributions of the melt pool. A stereovision technique is developed to acquire the height of the cladding. Based on the feedbacks, the scanning speed (worktable feeding speed), laser power, and powder delivery rate are regulated instantly. Based on many experiments and analyses, a number of metal parts have been formed by the intelligent MPLF system. These parts illustrate the capability of the system to fabricate fully-dense and near-net shaped parts.

All of the above-mentioned achievements pave the road towards an intelligent MPLF system. However, this multidisciplinary technology needs to be developed in many different aspects of technique to arrive at a fully functional intelligent MPLF machine. These techniques include the state-ofthe-art apparatus, advanced and knowledge-based controller, classic fabricating technics, and abundant experiment experience.

\section{REFERENCES}

[1] Zhang Kai, Liu Weiju, Shang Xiaofeng, (2005), Research on the processing experiments of laser metal deposition shaping, Optics \& Laser Technology, In press.

[2] J. Mazurnder, A. Scifferer, and J. Choi, (1999), Direct materials deposition: designed macro and microstucture, Material Research Innovation, vol. 3, no. 3, pp. 118-131.

[3] Radstok E, (1999), Rapid tooling, Rapid Prototyping Journal, Vol.5, No.4, pp. 164-169.

[4] Shang Xiaofeng, Liu Weijun, Wang Tianran,(2004), Laser Engineered Net Shaping Technology, Journal of Shanghai University, 8 (A02), pp. 26-29.

[5] Dongming Hu, Radovan Kovacevic, (2003), Sensing, modelling and control for laserbased additive manufacturing, Machine Tools and Manufacture, vol.43, pp.51-60.

[6] Guoqing Ning, et al, (2002), Research about close-loop control system during laser direct manufacturing metallic components, Applied Laser, vol.22, no.2.

[7] Yuichiro Tago, Funmie Akimoto, Kuniyuki Kitagawa, Norio Arai, (2004), Measurements of surface temperature and emissivity by two-dimensional four-color thermometry with narrow bandwith, ENERGY.

[8] J. Yen and R. Langarani, (1998), Fuzzy logic intelligence, control, and information, Prentice Hall, pp. 194-210.

[9] Liu Weijun, Dong Zaili, Hao Yingming, Zhu Feng, (2001), A robot self-location method based on stereovision system, High Technology Letters, pp.91-94, no.10. 